\title{
Dynamo-generated turbulence and outflows from accretion discs
}

\author{
BY AXEL BRANDENBURG \\ Department of Mathematics, University of Newcastle upon Tyne, \\ Newcastle upon Tyne NE1 7RU, UK and Nordita, Blegdamsvej 17, \\ DK-2100 Copenhagen Ø, Denmark
}

Local hydromagnetic simulations of accretion-disc turbulence currently provide the most convincing evidence that the origin of turbulence in discs could be the BalbusHawley magnetorotational instability. The main results of such calculations are highlighted with particular emphasis on the generation of large-scale magnetic fields. Comparison with mean-field dynamo theory is made. This theory is then used to address the question of the launching and collimation of winds emanating from the disc surfaces.

Keywords: magnetic fields; Balbus-Hawley instability; magnetorotational instability; alpha effect; jets; galaxy clusters

\section{Introduction}

In recent years, a number of hydromagnetic turbulence simulations have emerged that show the generation of large-scale fields of appreciable strength, often exceeding the equipartition field strength of the turbulence. This is possible if there is strong differential rotation from which to draw energy. One such example is in the context of accretion discs (Brandenburg et al. 1995), where the differential rotation is strong. Numerical simulations suggest that the magnetic energy can be several times larger than the turbulent kinetic energy. Another example is the geodynamo (Glatzmaier \& Roberts 1995), where the energy of the magnetic field strength exceeds the kinetic energy of the small-scale convective motions by several orders of magnitude. In the case of late-type stars, the magnetic energy is roughly comparable with the kinetic energy of the turbulence at the bottom of the convection zone, where the magnetic field can accumulate.

In the case of accretion discs, the turbulence is driven by the magnetic field itself through the magnetorotational (or Balbus-Hawley) instability (Balbus \& Hawley 1991). By contrast, in the case of the Sun, there is turbulent convection, which leads to dynamo action. However, the strong shear at the bottom of the convection zone can enhance the toroidal magnetic field to super-equipartition values. The strong toroidal magnetic field can become buoyantly unstable so that in those regions the flow is at least partly driven by the magnetic field, similar to the case of accretion discs. In the presence of rotation, magnetic buoyancy can, like thermal buoyancy, generate a poloidal field from a toroidal one and can thus cause an alpha-effect (Schmitt 1985; Brandenburg \& Schmitt 1998), which is important in mean-field dynamo theory (Krause \& Rädler 1980). 


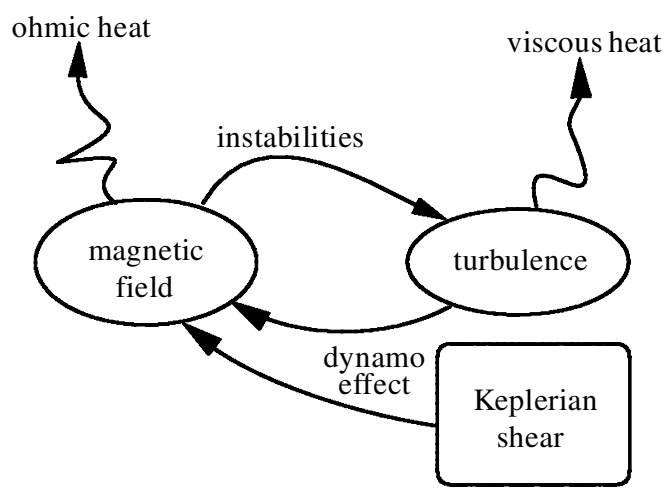

Figure 1. Sketch illustrating the flow of energy from shear energy into turbulent kinetic and magnetic energies, and, subsequently, into heat via ohmic and viscous heating.

Both in the Sun and in discs, the magnetic field extends beyond the actual dynamo region into the low-density exterior. There, the magnetic pressure can strongly exceed the gas pressure (small plasma beta) and the fluid motions are strongly affected by the magnetic field. Footpoint motions of the field lines anchored in the disc or the star are thought to bring field lines into complicated interwoven configurations that may reconnect once the magnetic stresses exceed some threshold. This is one of the possible processes that can heat the solar corona and may also contribute to heating the coronas of accretion discs. In both cases, the hot corona is unsteady and turns into a wind. In the case of discs, those outflows can take the form of a strong collimated jet.

The purpose of this paper is to review results and shortcomings of local accretiondisc simulations $(\S 2)$, and to assess what can be learned about the generation of large-scale magnetic fields in other bodies such as stars $(\S 3)$. The $\alpha-\Omega$ dynamo concept is then used to extrapolate our knowledge to systems with global geometry. In particular, we focus on the possibility of launching and collimating jets from the inner parts of an accretion disc without invoking an externally imposed large-scale magnetic field $(\S 4)$.

\section{Aspects of local accretion-disc simulations}

The Balbus-Hawley instability destabilizes laminar gas flows in differentially rotating bodies in the presence of an arbitrarily oriented magnetic field, provided the angular velocity decreases outwards and the field strength does not exceed some upper limit. The flows generated by this instability typically lead to turbulence (Hawley et al. 1995; Matsumoto \& Tajima 1995) and this turbulence has the property of enhancing and sustaining the magnetic field by dynamo action (Brandenburg et al. 1995; Stone et al. 1996). During this process, energy is drawn from the differential rotation. A major portion of the energy goes directly into magnetic energy. Part of it is directly dissipated via ohmic heating. The remaining part drives the turbulence, which is then also dissipated via viscous heating (see figure 1). It is possible that, although most of the energy goes first into magnetic energy, most of the dissipation is not magnetic but occurs through turbulent viscosity. In the original simulation of Brandenburg et al. (1995) the two terms were in fact comparable. 
The magnetic field in the disc could be maintained by currents outside the disc, for example in the central object (which could be a young star or a compact star) or in the environment in which the disc is embedded (molecular cloud or host galaxy). Another possibility, which will be discussed in more detail in the next section, is to generate and maintain the magnetic field within the disc itself by turbulent dynamo action. Before coming to that, we first address the important question of the strength of the kinetic and magnetic stresses that lead to an accretion flow. (This discussion applies to both dynamo-generated fields and externally maintained fields.) The total (kinetic and magnetic) stress is given by

$$
\left\langle\rho u_{\varpi}^{\prime} u_{\phi}^{\prime}-B_{\varpi}^{\prime} B_{\phi}^{\prime} / \mu_{0}\right\rangle
$$

where $\rho$ is the density, $\mathbf{u}$ the velocity, $\mathbf{B}$ the magnetic field, $\mu_{0}$ the magnetic permeability, and cylindrical polar coordinates $(\varpi, \phi, z)$ have been adopted. The angle brackets denote either horizontal or volume averages, and primes denote deviations from those averages. A convenient non-dimensional measure of the stress that is frequently adopted is

$$
\tilde{\alpha}_{\mathrm{SS}}=\left\langle\rho u_{\varpi}^{\prime} u_{\phi}^{\prime}-B_{\varpi}^{\prime} B_{\phi}^{\prime} / \mu_{0}\right\rangle /\langle p\rangle,
$$

where $p=\rho c_{\mathrm{s}}^{2}$ is the pressure and $c_{\mathrm{s}}$ is the isothermal sound speed. Numerical simulations of dynamo-generated turbulence carried out by various groups (Brandenburg et al. 1995, 1996a,b; Stone et al. 1996) suggest that the average value of $\alpha_{\mathrm{SS}}$ is ca. 0.01 if volume averages are adopted. Another somewhat different normalization, originally due to Shakura \& Sunyaev (1973), arises if the stress is expressed in terms of a turbulent viscosity, $\nu_{\mathrm{t}}=\alpha_{\mathrm{SS}} c_{\mathrm{s}} H$, where the stress is assumed to take a form similar to the viscous stress, albeit with a much larger (turbulent) viscosity, so

$$
\text { stress }=-\nu_{\mathrm{t}}\langle\rho\rangle \varpi \frac{\partial \Omega}{\partial \varpi},
$$

where $\Omega$ is the local angular velocity and $H$ is the disc height. In the above normalization with $\nu_{\mathrm{t}}=\alpha_{\mathrm{SS}} c_{\mathrm{S}} H$, we have $\alpha_{\mathrm{SS}}=0.47 \tilde{\alpha}_{\mathrm{SS}}$, where the numerical factor results from a $3 / 2$ factor for the Keplerian angular velocity gradient and a $\sqrt{2}$ factor from the definition of the scale height $H=\sqrt{2} c_{\mathrm{s}} / \Omega$. In this normalization, Brandenburg et al. (1996a) found $\alpha_{\mathrm{SS}} \approx 0.007$ in their highest-resolution calculations, which corresponds to $\tilde{\alpha}_{\mathrm{SS}} \approx 0.015$.

A problem with both representations is that the values of $\langle p\rangle$ and $\langle\rho\rangle$ decrease as the computational domain increases. At the same time, the kinetic and magnetic stresses do not strongly decrease with height (Brandenburg et al. 1996b). This makes the $\alpha_{\mathrm{SS}}$ value dependent on the size of the domain considered. This is avoided by expressing the stress in terms of $\Sigma \Omega c_{\mathrm{s}}$, where

$$
\Sigma=\int \rho \mathrm{d} z
$$

is the vertically integrated density. This yields a non-dimensional stress,

$$
\hat{\alpha}_{\mathrm{SS}}=\left\langle\rho u_{\varpi}^{\prime} u_{\phi}^{\prime}-B_{\varpi}^{\prime} B_{\phi}^{\prime} / \mu_{0}\right\rangle /\left(\Sigma \Omega\left\langle c_{\mathrm{s}}\right\rangle\right),
$$

that is, in our present case, approximately four times smaller than $\tilde{\alpha}_{\mathrm{SS}}$, because $\Sigma=\langle\rho\rangle L_{z}$, and the vertical box size, $L_{z}$, is approximately four scale heights, so $\hat{\alpha}_{\mathrm{SS}}=0.002$. 

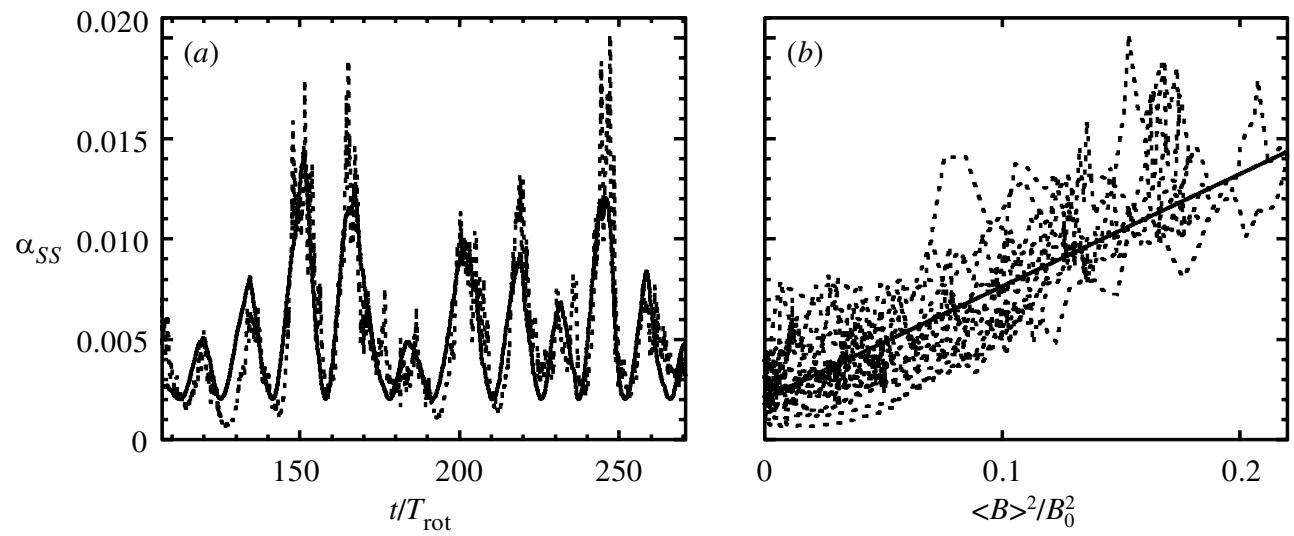

Figure 2. Dependence of $\alpha_{\mathrm{SS}}$ on time and the mean magnetic field strength. Here, $B_{0}=$ $\left\langle\mu_{0} \rho c_{\mathrm{s}}^{2}\right\rangle^{1 / 2}$ is the thermal equipartition field strength and $T_{\text {rot }}=2 \pi / \Omega$ is the local rotation period. (a) The dotted line represents the actual data and the solid line gives the fit obtained by correlating $\boldsymbol{\alpha}_{\mathrm{SS}}$ with the mean magnetic field $(b)$.

It is important to realize that the value of $\alpha_{\mathrm{SS}}$ changes significantly with time. This is mostly because the dynamo-generated magnetic field varies strongly with time. In figure 2 we plot the dependence of $\alpha_{\mathrm{SS}}$ on time and on the mean toroidal magnetic field strength.

It is quite conceivable that the field strength resulting from the local dynamo may not be representative of the field that would be present in global geometry. In local geometry with periodic (or shearing-periodic) boundary conditions, the vertical mean magnetic field is strictly zero at all times. It is quite possible that the overall field strength will increase once the vertical mean field is no longer restricted to be zero, as in local simulations. Nevertheless, a potentially important result from the local simulations is that for both dynamo-generated fields and for imposed fields, $\alpha_{\mathrm{SS}}$ is proportional to the instantaneous mean toroidal field strength, so

$$
\alpha_{\mathrm{SS}} \approx 0.12\langle\mathbf{B}\rangle^{2} /\left\langle\mu_{0} \rho c_{\mathrm{S}}^{2}\right\rangle
$$

(see Brandenburg (1999), correcting an error in the coefficient in Brandenburg et al. $(1996 a))$. One may hope that this dependence remains approximately valid even in the global case. If so, and if the mean magnetic energy becomes comparable with the thermal energy, one may expect that in the global case the values of $\alpha_{\mathrm{SS}}$ could be significantly larger than the value of 0.01 obtained so far.

\section{Magnetic field regeneration}

\section{(a) The dynamo in disc simulations}

We now discuss properties of the dynamo-generated field. Unlike the dynamo simulations of Hawley et al. (1996) with periodic boundary conditions in the vertical direction, in the simulations of Brandenburg et al. (1995), where the horizontal field components were assumed to vanish on the top and bottom boundaries, the toroidal magnetic flux was not constrained to vanish. Even though the initial field had zero net flux, after some time the horizontal net flux, or mean field, became significantly different from zero. This mean field varied approximately periodically. 

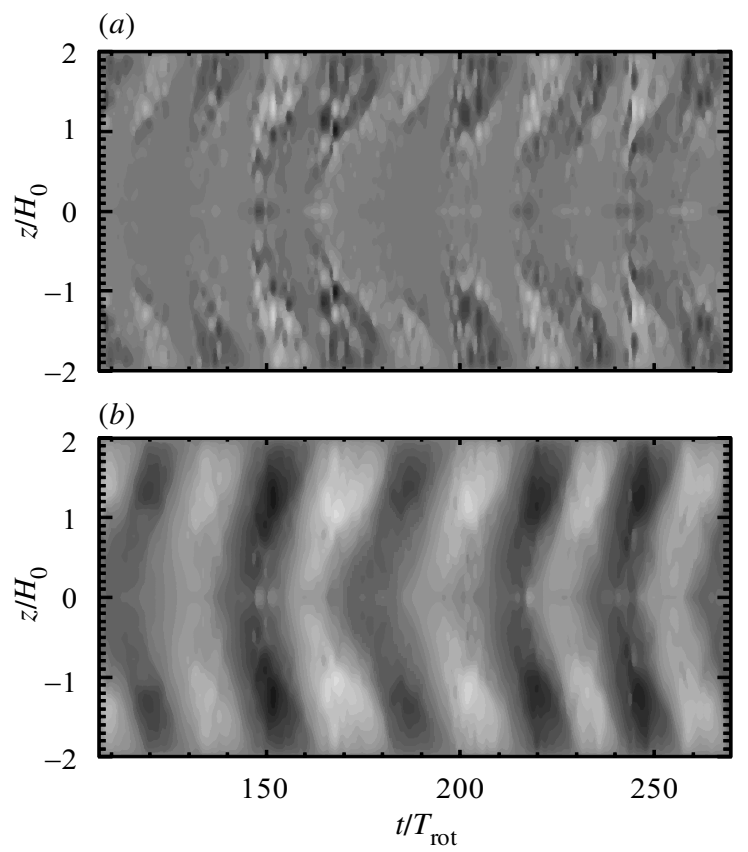

Figure 3. Butterfly (space-time) diagram of the poloidal and toroidal magnetic field components averaged over the two horizontal $(x$ - and $y$-) directions. Note that the poloidal field is much more noisy than the toroidal field, and that there is a clear outwards migration of magnetic field.

Throughout this section, we use a local Cartesian box that is located at position $(\varpi, \phi, z)=\left(R, R \Omega_{0}, 0\right)$, where $\Omega_{0}=\sqrt{G M / R^{3}}$ and $H / R \ll 1$. Our Cartesian coordinates are $(x, y, z)$, where $x=\varpi-R$ and $y=R\left(\phi-\Omega_{0} t\right)$.

In figure 3 we plot a space-time diagram of the mean poloidal and toroidal magnetic fields, $\left\langle B_{x}\right\rangle$ and $\left\langle B_{y}\right\rangle$, respectively, as functions of $t$ and $z$, where time $t$ is measured in units of the orbital period, $T_{\text {rot }}=2 \pi / \Omega$, and height $z$ above the midplane is measured in units of the initial vertical density scale height $H_{0}$. In this particular simulation, the magnetic field is enforced to be strictly symmetric about the midplane. Without this restriction the field turned out to be very nearly symmetric about the midplane (see Brandenburg et al. 1995). We note that $\left\langle B_{x}\right\rangle$ is approximately 30 times weaker and much more noisy than $\left\langle B_{y}\right\rangle$, but in both cases there is a clear spatio-temporal structure with a finite migration speed away from the midplane to the upper and lower boundaries, $z= \pm 2 H_{0}$. The initial scale height, $H_{0}$, differs somewhat from the actual scale height because of viscous and ohmic heating, which cause the temperature in the disc to increase.

The fact that a large-scale magnetic field is generated is interesting, because it demonstrates the ability of the system to produce magnetic fields on scales larger than the typical scale of the turbulence, but comparable with the size of the box. This, on the other hand, also means that the size, geometry and other properties of the box, such as boundary conditions, do matter. The limitations of a local simulation are therefore clear: in order to get things right, one really has to simulate the whole system covering a much larger range of scales. The only turbulence simulation to date which attempted to do this was done by Armitage (1998), but there was no vertical 

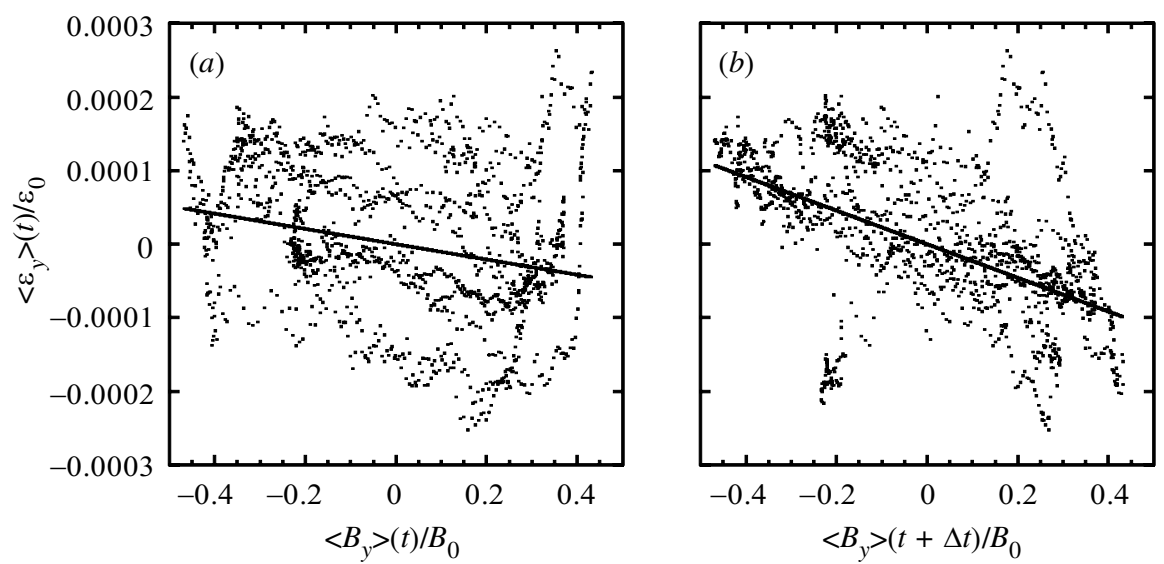

Figure 4. Correlation of the mean toroidal component of the electromotive force with the mean toroidal magnetic field. Here, the $\left\langle B_{y}\right\rangle$ and $\mathcal{E}_{y}$ fields are averaged over the entire Northern Hemisphere and normalized with $B_{0}=\left\langle\mu_{0} \rho c_{\mathrm{s}}^{2}\right\rangle^{1 / 2}$ and $\mathcal{E}_{0}=\left\langle c_{\mathrm{s}}\right\rangle B_{0}$, respectively. The negative correlation is suggestive of a negative $\alpha$ effect. Note, however, the strong noise in the relation between $\mathcal{E}_{y}$ and $\left\langle B_{y}\right\rangle$. The noise is somewhat reduced when $\mathcal{E}_{y}$ is correlated with $\langle B\rangle_{y}$ at a later time, $t+\Delta t$, where $\Delta t=5 T_{\text {rot }}$, corresponding to a $\pi / 3$ phase lag $(b)$.

stratification and there was also no large-scale field on the scale of the entire disc. This lack of large-scale coherence of the field could be explained on the one hand simply by the fact that the simulation has not been run for long enough. On the other hand, this lack of large-scale field coherence could be connected with the absence of stratification in this case. It is well known that the combination of stratification and rotation leads to a systematic departure from mirror-symmetry, as measured by helicity for example. This is known to be a crucial prerequisite of $\alpha-\Omega$ dynamos (e.g. Krause \& Rädler 1980), which will be discussed next.

A conceptual difficulty in applying $\alpha-\Omega$ dynamos to discs is that the turbulent flow is itself driven by the magnetic field. Normally the $\alpha-\Omega$ dynamo is used to explain the amplification of weak seed magnetic fields for a given turbulence field. Here, on the other hand, the turbulence is already magnetized. Indeed, there have been several approaches to determine an $\alpha$ effect in the presence of a small-scale magnetic field. Frisch et al. (1975) and Pouquet et al. (1976) found that $\alpha$ is to be replaced by the residual $\alpha$, which consists of kinetic and current helicity. Similar ideas go back to Vainshtein (1972) and Zeldovich et al. (1983). However, in our case, the turbulence and the small-scale magnetic field are highly anisotropic, so $\alpha$ must be a highly anisotropic tensor. Since shear is very strong, the $\alpha$ effect can be neglected for generating a mean toroidal magnetic field. (We assume here that the mean field is axisymmetric; otherwise this approximation would not be valid.) However, the poloidal (or radial) field cannot be generated by shear, but it can be regenerated from toroidal field by the phi-phi component of the $\alpha$ tensor. In our Cartesian model, we refer to this component as $\alpha_{y y}$.

\section{(b) Evidence for a dynamo $\alpha$ from the simulations}

The $\alpha$ tensor relates the mean electromotive force to the mean magnetic field. In order to assess the possibility of such a relation, in figure 4 we plot the correlation of 
the mean toroidal field with the toroidal and radial components of the electromotive force, $\mathcal{E}=\left\langle\mathbf{u}^{\prime} \times \mathbf{B}^{\prime}\right\rangle$.

Looking at figure 4 , one can see that $\mathcal{E}$ is correlated with the horizontal components of $\langle\mathbf{B}\rangle$. The proportionality coefficient between $\mathcal{E}_{y}$ and $\left\langle B_{y}\right\rangle$ is an important quantity and is basically our $\alpha_{y y}$. This $\alpha$ is not to be confused with the Shakura-Sunyaev alpha, which carries the subscript SS. Note that $\alpha_{y y}$ is negative in the upper disc plane. This is surprising in view of the fact that conventional theories that assume isotropy (e.g. Moffatt 1978) predict $\alpha_{y y}$ to be a negative multiple of the kinetic helicity, which, in our case, has a negative sign, as expected for cyclonic turbulence (e.g. Parker 1979). The unconventional sign of $\alpha_{y y}$ can be explained if the flow is governed by magnetic (instead of thermal) buoyancy in the presence of strong shear and rotation (Brandenburg 1997, 1999). This idea has now also been verified using the first-order smoothing approach (G. Rüdiger, personal communication).

It is clear that the $\alpha$ effect is actually extremely noisy. We note that the strong scatter can be somewhat reduced if a $\pi / 3$ phase lag of the magnetic field is assumed (see figure $4 b$ ), which could be suggestive of a non-instantaneous or dynamical $\alpha$ effect (see, for example, Kleeorin et al. 1995; Covas et al. 1997). Nevertheless, the correlation between $\langle\mathbf{B}\rangle$ and $\mathcal{E}$ remains relatively loose. The possibility of strong fluctuations has prompted Vishniac \& Brandenburg (1997) to investigate the dynamo effect in this case where $\alpha$ is changed randomly as a function of space and time. They referred to this as the incoherent $\alpha$ effect. In the absence of shear, Moffatt (1978) found that for a random (incoherent) $\alpha$ effect, the magnetic field can grow at the smallest possible scale (a negative magnetic diffusion effect). However, the shear, together with turbulent diffusion, can cause the toroidal field to have the same sign over the entire disc height for times comparable with the magnetic diffusion time. This could be the reason why the mean toroidal field varies reasonably smoothly in space and time, in spite of an extremely noisy $\alpha$ effect.

If anything, it is probably a combination of incoherent and coherent (ordinary) $\alpha$ effect that could be invoked to explain the mean-field generation in our simulation. Otherwise, with the incoherent $\alpha$ effect alone, it would be difficult to explain the systematic migration away from the midplane, which is a natural property of the migratory Parker dynamo with negative $\alpha$ effect in the upper disc plane and a negative angular velocity gradient.

\section{(c) Comparison with predictions from $\alpha-\Omega$ dynamo theory}

Here, we discuss a number of aspects of mean-field $\alpha-\Omega$ dynamos that can be compared with properties of the actual turbulence simulation. We adopt horizontal averages that are functions of $z$ and $t$. This is appropriate to characterize the local disc simulations. However, because the horizontal boundaries are periodic, the vertical flux, i.e. the vertical mean field, is constant in time. Furthermore, because $\nabla \cdot\langle\mathbf{B}\rangle=$ $\partial\left\langle B_{z}\right\rangle / \partial z=0,\left\langle B_{z}\right\rangle$ is also constant in $z$. Since $\left\langle B_{z}\right\rangle=0$ initially, $\left\langle B_{z}\right\rangle=0$ for all times. Thus, $\langle\mathbf{B}\rangle=\left(\left\langle B_{x}\right\rangle,\left\langle B_{y}\right\rangle, 0\right)$. The $\alpha-\Omega$ dynamo equations are then

$$
\begin{aligned}
\frac{\partial}{\partial t}\left\langle B_{x}\right\rangle & =-\frac{\partial}{\partial z}\left(\alpha_{y y}\left\langle B_{y}\right\rangle\right)+\eta_{\mathrm{t}} \frac{\partial^{2}}{\partial z^{2}}\left\langle B_{x}\right\rangle, \\
\frac{\partial}{\partial t}\left\langle B_{y}\right\rangle & =-\frac{3}{2} \Omega\left\langle B_{x}\right\rangle+\eta_{\mathrm{t}} \frac{\partial^{2}}{\partial z^{2}}\left\langle B_{y}\right\rangle,
\end{aligned}
$$

Phil. Trans. R. Soc. Lond. A (2000) 
where we have assumed that $\eta_{\mathrm{t}}=$ const., which is not a very crucial simplification. The onset of dynamo action is governed by the dynamo number, $D=\frac{3}{2} \alpha_{0} \Omega H_{0}^{3} / \eta_{\mathrm{t}}^{2}$, where $\alpha_{y y}=\alpha_{0} z / H_{0}$ has been assumed. See Brandenburg (1999) for details. We are mostly concerned with the case $\alpha_{0}<0$; the critical dynamo number is then $D \approx-400$.

We now highlight four points where agreement between simulations and mean-field model can be found.

(i) The most easily excited solution is oscillatory and has quadrupole-type parity. This agrees with the behaviour and parity of the mean field found in the simulations. Furthermore, if perfectly conducting boundaries are assumed, i.e. $\partial\left\langle B_{x}\right\rangle / \partial z=\partial\left\langle B_{y}\right\rangle / \partial z=0$ on the boundaries, the mean-field model predicts steady solutions with dipole-type parity (again for $\alpha_{0}<0$ ). This too is in agreement with the original simulations (see Brandenburg 1999).

(ii) For oscillatory solutions, the migration direction of the field depends on the sign of $\alpha_{0}$. Only for $\alpha_{0}<0$ do we have migration away from the midplane. This is in agreement with the simulation, where the magnetic field does move away from the midplane and where a direct determination of $\alpha_{0}$ suggests negative values (see figure 4).

(iii) Mean-field theory predicts that the magnetic cycle period is related to the magnitude of $\alpha_{0}$ via

$$
T_{\text {cyc }} / T_{\text {rot }}=\left|\alpha_{0} / \Omega H\right|^{-1 / 2}
$$

The simulation gave $\alpha_{0} \approx-0.001 \Omega H$, so $T_{\text {cyc }} \approx 30 T_{\text {rot }}$, which is in good agreement with the simulation; see figure 3 to verify that $T_{\text {cyc }} \approx 30 T_{\text {rot }}$.

(iv) The value of $\eta_{\mathrm{t}}$ can be estimated by assuming that the mean-field dynamo is marginally excited. This yields $\eta_{\mathrm{t}} \approx 0.008 \Omega H^{2}$. Assuming, furthermore, a turbulent magnetic Prandtl number of unity, this would correspond to a value of $\nu_{\mathrm{t}}$ that is close to the value obtained from the horizontal stress (see $\S 2$ ).

Although the similarities between the $\alpha-\Omega$ model and simulation are remarkable, one must realize that there are still some major problems with $\alpha-\Omega$ theory. We recall that the simulations give extremely noisy values of $\alpha$, and this may become worse at higher resolution. The magnetic Reynolds numbers are around 100. For larger values, it takes longer before the simulations start to produce magnetic fields with large-scale spatio-temporal coherence. Also, attempts to verify the detailed functional dependence of $\mathcal{E}(\langle\mathbf{B}\rangle)$ suggest that the product $\alpha\langle\mathbf{B}\rangle$ may need to be replaced by a convolution, $\alpha \star\langle\mathbf{B}\rangle$, which corresponds to a multiplication in Fourier space. An analysis of the simulation data seems to suggest that the contributions from the smallest wavenumbers dominate (Brandenburg \& Sokoloff 2000). In that sense, a one-mode truncation of the dynamo equations could possibly be closer to the truth than the original partial differential equation. Similar suggestions have been made in the context of stellar cycles (Brandenburg et al. 1998).

A more detailed test of $\alpha-\Omega$ dynamo theory would be to compare dynamos in global geometry. It is therefore important to produce a global simulation of a turbulent disc dynamo with stratification included. There are still some major difficulties, especially in connection with the low-density regions away from the midplane. Of 
great astrophysical interest, of course, is the investigation of associated outflows that are expected to be collimated into a jet at larger distances above the disc. In the following, we explore this question using a two-dimensional axisymmetric model, where the magnetic field is generated by an $\alpha-\Omega$ dynamo with negative $\alpha$ effect in the upper disc plane.

\section{Outflows from disc dynamos}

We now want to discuss the possible role of dynamo-generated magnetic fields for launching and collimating jets emanating from accretion discs. $\dagger$ Our main motivation for this type of work stems from the somewhat unsatisfactory feature of many jet models, that a prescribed axial magnetic field has to be assumed (e.g. Bell \& Lucek 1995; Matsumoto et al. 1996; Ouyed et al. 1997; Ouyed \& Pudritz 1997; Ustyugova et al. 1999). In star-forming regions, such as in NGC 1333, a large number of jets, apparently all pointing in different directions, are seen (Hodapp \& Ladd 1995). This would make the idea of a uniformly directed large-scale field as the main collimator perhaps less plausible. It is possible, however, that differently aligned jets are far apart in the direction along the line of sight. Also, even though the star-forming process is not uniform, an initially non-uniform magnetic field could still end up being coherent over the length of the jet. However, in the case of jets emanating from X-ray binaries, such as microquasars (Mirabel \& Rodriguez 1998), the disc material comes from a secondary, so there is no external source of magnetic flux to replenish the field that is dragged inwards. Also, it is difficult to envisage how, in that case, an imposed field could be compressed to field strengths that are large enough to be dynamically important for collimating the jet.

Our model consists of a prescribed disc in which a non-vanishing $\alpha$ effect and a nonvanishing turbulent diffusivity are assumed. The $\alpha$ coefficient is negative in the upper disc plane. In this case, the magnetic field would be oscillatory and of quadrupolar parity (i.e. symmetric about the midplane), but the outflow can turn the field into a steady dipolar one (antisymmetric about the midplane). Furthermore, we assume that the model is piecewise polytropic such that the entropy in the disc is lower than in the halo surrounding the disc. The initial state is an equilibrium solution where the halo is gas-pressure supported, but the disc, which is cooler and denser than the halo, is supported mainly centrifugally. We simulate the cooler disc here by a fixed entropy profile. This is done as a crude substitute for the missing radiation and ionization physics that is responsible for cooling the surfaces of star and disc. We inject matter locally into the disc using a source term in the continuity equation, which is different from zero only where the density in the disc drops below its initial value. This source term is proportional to the density deficit. There is a corresponding term in the momentum equation to ensure that the matter is injected at the local Keplerian speed. Again, the motivation behind this approach is to represent some of the physics of the underlying, much denser, disc, where most of the material is supplied from much larger radii. Our simulation is only able to represent the lessdense layers away from the midplane, and any removal of mass there must be quickly replenished by hydrostatic adjustment within the disc.

$\dagger$ In this section, we report preliminary work that was done in collaboration with Wolfgang Dobler, Anvar Shukurov and Brigitta von Rekowski, all from Newcastle.

Phil. Trans. R. Soc. Lond. A (2000) 
(a)

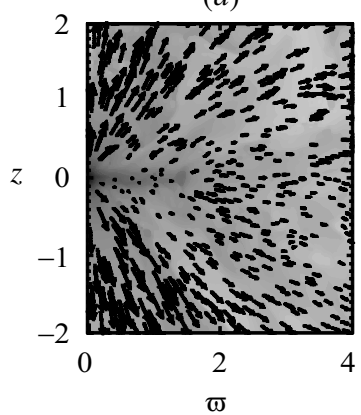

(b)

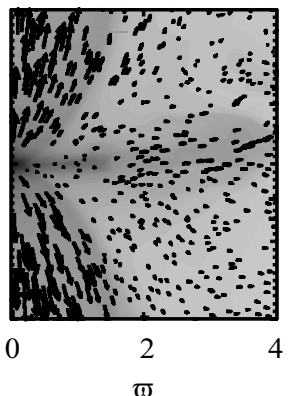

(c)

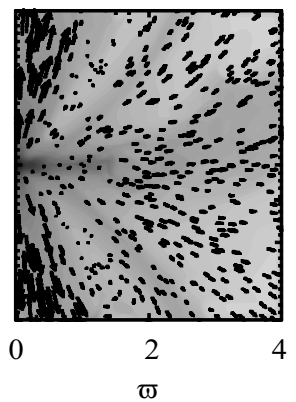

Figure 5. Evolution of poloidal velocity field and logarithmic density (greyscale coded background: dark means high density).

The hydromagnetic equations are solved in cylindrical polar coordinates, $(\varpi, \phi, z)$, assuming axisymmetry. Spatial derivatives are calculated using sixth-order finite differences, and a third-order Runge-Kutta integrator has been adopted. A shockcapturing viscosity proportional to the local compression has been used.

In figure 5 we give a sequence of snapshots showing how an outflow is produced. At first this outflow transports angular momentum and toroidal magnetic field at an angle of $c a .45^{\circ}$ from the disc. As time goes on, the toroidal magnetic field in the halo and away from the axis begins to grow, both because of magnetic buoyancy and advection from the disc and because of shear. Eventually, the magnetic pressure from the toroidal magnetic field in the outer parts becomes comparable with the gas pressure on the axis. This is the time when the jets begin to collimate.

In figure 6 we plot field lines together with velocity vectors on a background representing the density (dark means high density). Near the disc surface, the field lines diverge radially from the centre, but further away from the disc, the field lines that are close to the axis become vertical. There is a converging cone around the axis where the density is enhanced. Here, the wind speed is highest. This is because those field lines originate from a point close to the star, where the gravitational potential is deepest, so the escape velocity for particles on those field lines is highest.

The field lines that are further away from the axis originate from cooler parts of the disc and the outflow along those field lines is slower, but still very important, because this slow wind transports significant amounts of toroidal magnetic field and angular momentum from the disc into the outer parts of the halo.

In order to see where the mass actually comes from, in figure 7 we plot a close-up of the inner part of the disc. Here, the greyscale-coded background represents the density source term. Dark shades represent large local mass injection. Note that the strongest mass injection is not where most of the disc material goes into the wind and where the jets emanate from. Instead, the strongest mass source in the disc is at intermediate radii. The injected mass flows first through the inner parts of the disc towards the centre. The resulting overpressure near the centre then drives the outflow.

There are a number of characteristic features of this jet model:

(i) the jet is hot and dense compared with the regions away from the axis;

(ii) the jet has very little angular momentum; and

(iii) the jet is superfast, i.e. its speed exceeds the fast magnetosonic speed. 


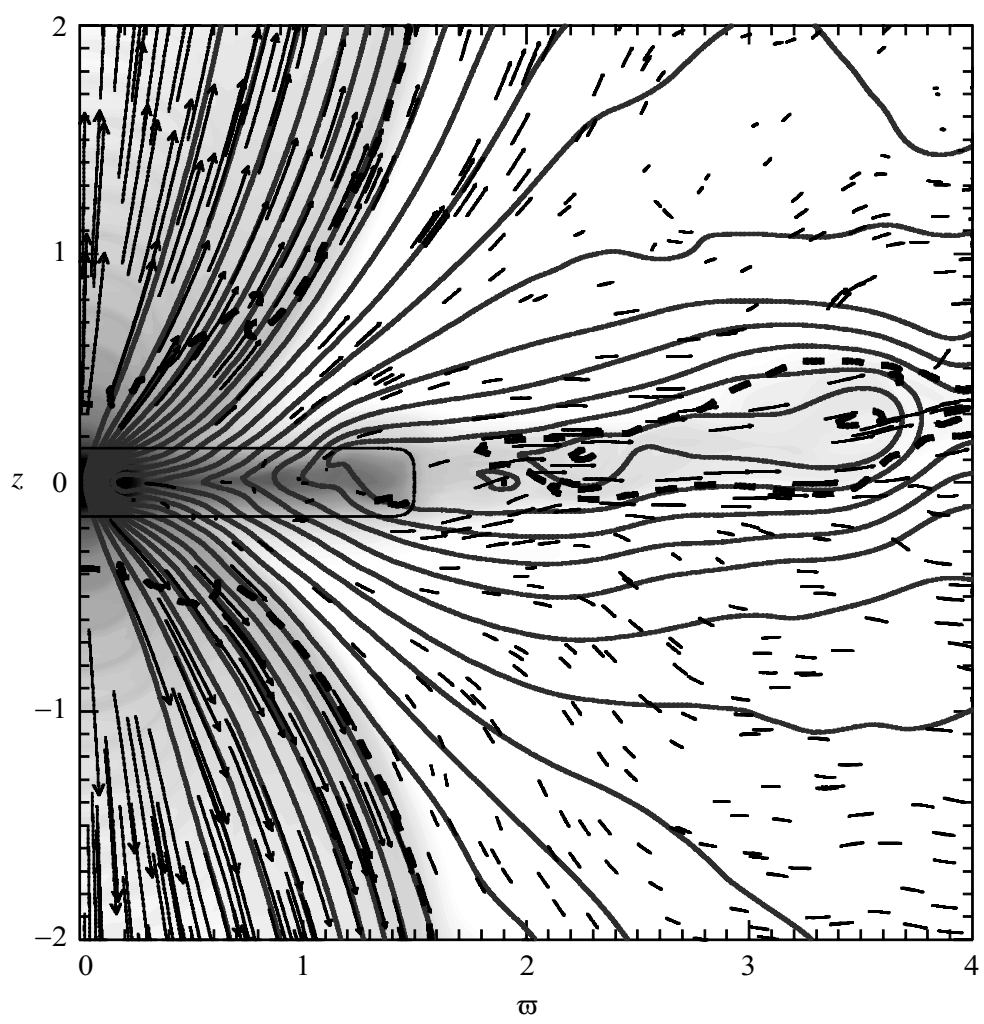

Figure 6. Poloidal velocity vectors and poloidal magnetic field lines superimposed on a greyscale image of the logarithmic density. Dark means high density. The thick dashed line denotes the location where the poloidal flow speed equals the fast magnetosonic speed. The thin solid line gives the location of the disc surface. The slight asymmetry in the field is a relic from the mixed-parity initial condition.

The jet presented here is not centrifugally driven, as one might have expected. However, in the outer regions of the disc, the magnetic field is tilted sufficiently far away from the axis that centrifugal acceleration does occur. However, the material accelerated in that way does not directly contribute to the jet, but rather to the exterior of the jet, which is continuously gaining toroidal magnetic field that contributes to the collimation once the magnetic pressure becomes comparable with the gas pressure in the jet (figure 8).

Looking at the temperature (or enthalpy $h=c_{p} T$ ) profile along the jet axis (figure 9) one sees that the temperature is just slightly smaller than the initial (virial) temperature, which corresponds to the hydrostatic state (dotted line). Thus, the thermal energy in the jet is comparable with the potential energy, so

$$
T_{\text {jet }}(z) \lesssim T_{\text {vir }}=\left(1-\frac{1}{\gamma}\right) \frac{\mu}{\mathcal{R}} \frac{G M}{z},
$$

where $\mathcal{R}$ is the universal gas constant, $\mu$ the mean molecular weight, $G$ the gravitational constant, and $M$ the mass of the central object. Table 1 gives the temperature for some relevant values of $z$ and for $M=1 M_{\odot}$ and $10^{8} M_{\odot}$. 


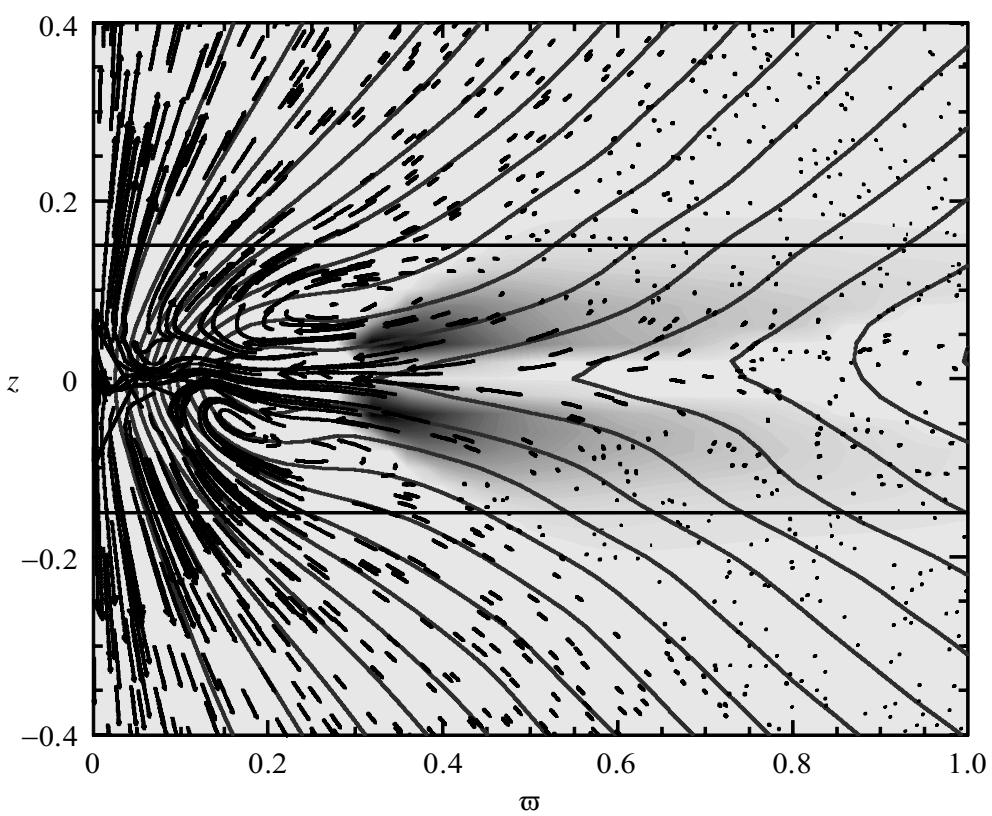

Figure 7. Close-up of the inner parts of the disc. Poloidal mass flux vectors and poloidal magnetic field lines superimposed on a greyscale image of the local rate of mass injection, i.e. the source term in the continuity equation.

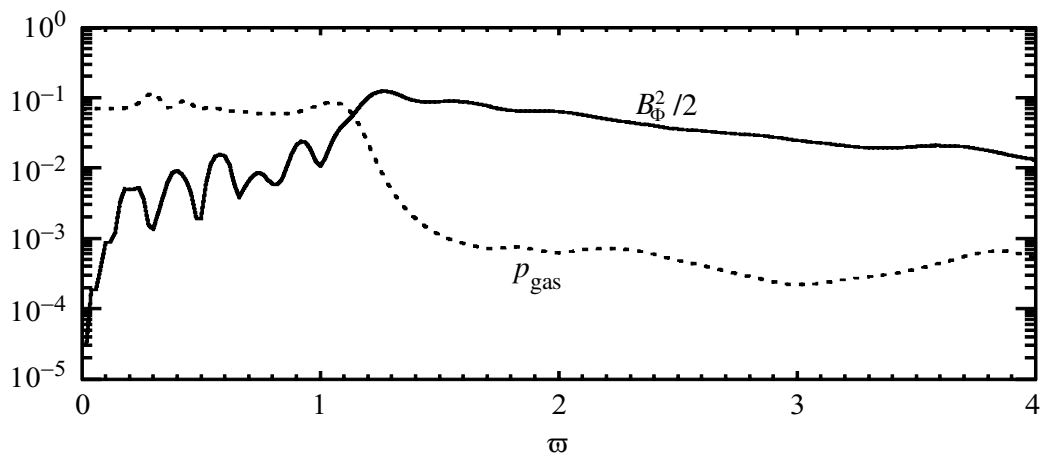

Figure 8. Radial cross-section through $z=1$ showing gas and magnetic pressures. Note that for $\varpi \geqslant 1$, the magnetic pressure dominates over the gas pressure by two orders of magnitude. The radial magnetic pressure gradient at $\varpi \approx 1$ provides the main confinement of the jet.

At large distances where jets have been observed (ca.100 AU for protostellar jets and $c a .10 \mathrm{kpc}$ for galactic jets), the jet temperature does not, therefore, appear to be much larger than the observational limits. A full account of those results is to be published elsewhere (Brandenburg et al. 2000).

\section{Discussion}

The purpose of this review has been to highlight the importance of magnetic fields in driving various types of activity. While stellar activity could have been considered 

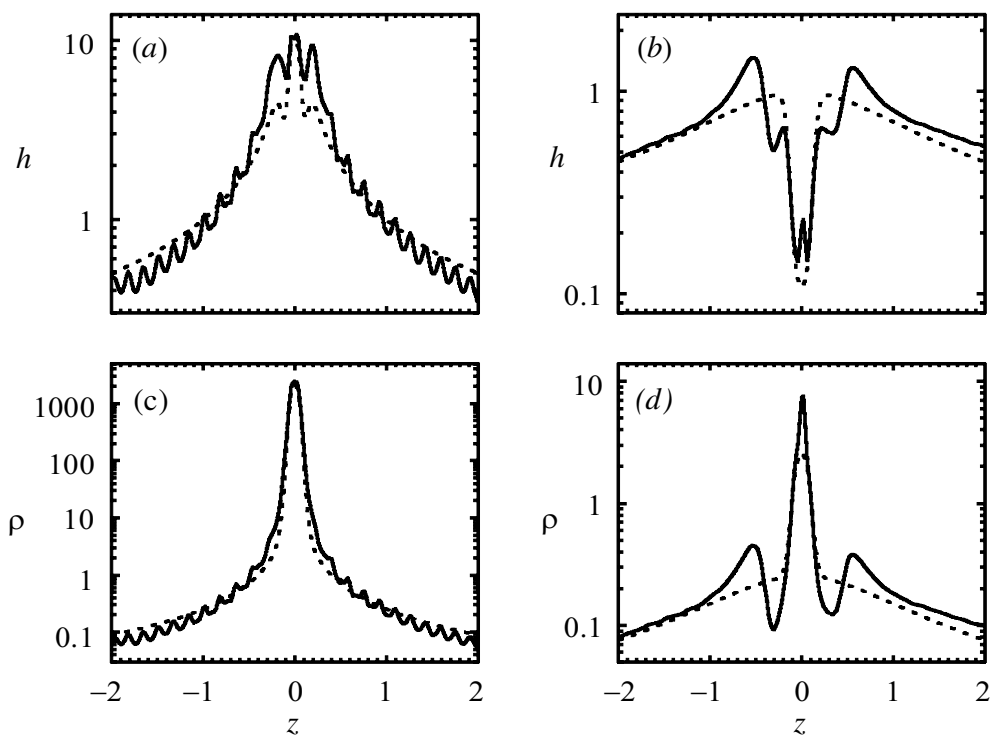

Figure 9. Vertical enthalpy profiles through $\varpi=0$ (i.e. through the star) and $\varpi=0.8$ (i.e. through the disc). The waves in the halo are numerically well resolved, but their excitation is probably an artefact of the instantaneous response of the disc to local mass loss.

Table 1. Virial temperatures for different distances from (i) a protostellar object of one solar mass and (ii) an active galactic nucleus with a supermassive black hole of $10^{8}$ solar masses (For the mean molecular weight we assumed $\mu=0.6$, except when $T<5000 \mathrm{~K}$ when $\mu=1.2$ was taken.)

\begin{tabular}{ccc}
\hline$z$ & $T_{\text {vir }}\left(M=1 M_{\odot}\right)(\mathrm{K})$ & $T_{\text {vir }}\left(M=10^{8} M_{\odot}\right)(\mathrm{K})$ \\
\hline $0.01 \mathrm{AU}$ & $3 \times 10^{6}$ & \\
$1 \mathrm{AU}$ & $3 \times 10^{4}$ & $3 \times 10^{12}$ \\
$100 \mathrm{AU}$ & $5 \times 10^{2}$ & $3 \times 10^{10}$ \\
$1 \mathrm{pc}$ & & $1 \times 10^{7}$ \\
$10 \mathrm{kpc}$ & & $3 \times 10^{3}$ \\
\hline
\end{tabular}

secondary in the sense that one first has to understand stellar structure, this is no longer true in the case of accretion discs. The turbulence in discs is now believed to be driven by a magnetic instability, so, if there was no magnetic field, there would be no turbulence and no turbulent dissipation, and, hence, no radiation.

Magnetic fields are also important for jets. The field contributes to the launching of the jet (at least from some parts of the disc) and is probably important for collimating it. The outflows, on the other hand, provide an important channel for bringing material from the stellar disc to the interstellar medium, or from galaxies into the intergalactic cluster gas, as is shown by the high metallicities in clusters. $\dagger$ Our simulations imply a significant amount of magnetic field injection. Preliminary estimates suggest that the surface-integrated Poynting flux is $c a .10 \%$ of the surface integrated kinetic flux, which is roughly $\dot{M}_{\mathrm{w}} c_{\mathrm{s}}^{2}$, where $\dot{M}_{\mathrm{w}}$ is the mass-loss rate from outflows

$\dagger$ I thank Åke Nordlund for attracting my attention to this possibility.

Phil. Trans. R. Soc. Lond. A (2000) 
or winds in general, $c_{\mathrm{s}}$ is the sound speed (or possibly the fast magnetosonic speed) at the point where the flow becomes transonic (or superfast). Applied to the case of galaxy clusters with $c a .10^{4}$ galaxies, each with $\dot{M}_{\mathrm{w}} \approx 0.1 M_{\odot} \mathrm{yr}^{-1}=10^{25} \mathrm{~g} \mathrm{~s}^{-1}$, and $c_{\mathrm{s}}=1000 \mathrm{~km} \mathrm{~s}^{-1}$ for the sound speed in the intracluster gas, we find that the rate of magnetic energy injection for all galaxies together is $L_{\mathrm{mag}}=10^{44} \mathrm{erg} \mathrm{s}^{-1}$. Distributing this over the volume of the cluster of $V \sim 1 \mathrm{Mpc}^{3}$, and integrating over a duration of $\Delta t=1 \mathrm{Gyr}$, this corresponds to a mean magnetic energy density of $\left\langle\mathbf{B}^{2} / 8 \pi\right\rangle \approx 10^{-13} \mathrm{erg} \mathrm{cm}^{-3}$, so

$$
\left\langle\mathbf{B}^{2}\right\rangle^{1 / 2} \approx\left(8 \pi \frac{F_{\text {Poy }}}{F_{\text {kin }}} \frac{N \dot{M}_{\mathrm{w}} c_{\mathrm{s}}^{2}}{V} \Delta t\right)^{1 / 2} \approx 10^{-6} \mathrm{G},
$$

which is indeed of the order of the field strength observed in galaxy clusters. Similar values are obtained when considering outflows from stellar discs. We note that our estimate has been rather optimistic in places $\left(\dot{M}_{\mathrm{w}}\right.$ could be lower, or the relevant $\Delta t$ could be shorter, for example), but it does show that outflows are bound to produce significant magnetization of the intracluster gas and the interstellar medium (see, also, Völk \& Atoyan 1999). In the latter case, it will provide a good seed field for the galactic dynamo. A dynamo is still necessary to shape the magnetic field and to prevent if from decaying in the galactic turbulence. Similarly, many galaxy clusters undergo merging and this too can enhance and reorganize the magnetic field.

There are many open questions that need to be addressed in the near future. There is now an urgent need to begin global simulations of the turbulent disc. As we have seen from the mean-field calculations the properties of the large-scale field will be affected by boundary conditions and also, therefore, by the presence of an outflow. Thus, full allowance for an outflow should be made in such global calculations. Also, ionization and radiation processes should be included at some point to produce a realistic temperature and entropy stratification in the disc and the lower corona. Another line of related research is to simulate the actual formation of a disc with its associated outflows all the way from the initial collapse. This could be done both for protostars and for protogalaxies, although the detailed physics is different in those two cases. Another, perhaps simpler, problem is the study of discs in mass-exchanging binaries, because there the mass supply is sustained and the disc is, therefore, more nearly stationary on longer time-scales.

Another important problem that needs to be clarified is whether the magnetic field that collimates the jet can be considered given, or whether it is generated within the disc. While it is conceivable that the field strength achieved during the collapse could be strong enough, there does not seem to be a process that could lead to appreciable field strengths in the case of mass-exchanging binaries, such as microquasars (see Mirabel, this issue). Thus, the model presented in the previous section, in which the field was generated solely within the disc by dynamo action, could be more relevant to this case.

It is a pleasure to thank Åke Nordlund and Anvar Shukurov for stimulating discussions, and Wolfgang Dobler and Brigitta von Rekowski for developing the code. I am also grateful to David Moss, Rachid Ouyed and Nigel Weiss for useful comments on the manuscript. This work was supported in part by the PPARC grant GR/L30268.

Phil. Trans. R. Soc. Lond. A (2000) 


\section{References}

Armitage, P. J. 1998 Turbulence and angular momentum transport in global accretion disk simulation. Astrophys. J. 501, L189-L192.

Balbus, S. A. \& Hawley, J. F. 1991 A powerful local shear instability in weakly magnetized disks. I. Linear analysis. Astrophys. J. 376, 214-222.

Bell, A. R. \& Lucek, S. G. 1995 Magnetohydrodynamic jet formation. Mon. Notes R. Astr. Soc. 277, 1327-1340.

Brandenburg, A. 1997 Large scale turbulent dynamos. Acta Astron. Geophys. Univ. Comenianae XIX, 235-261.

Brandenburg, A. 1999 Disc turbulence and viscosity. In Theory of black hole accretion discs (ed. M. A. Abramowicz, G. Björnsson \& J. E. Pringle), pp. 61-86. Cambridge University Press.

Brandenburg, A. \& Schmitt, D. 1998 Simulations of an alpha-effect due to magnetic buoyancy. Astron. Astrophys. 338, L55-L58.

Brandenburg, A. \& Sokoloff, D. 2000 Local and nonlocal magnetic diffusion and alpha-effect tensors in shear flow turbulence. Geophys. Astrophys. Fluid Dyn. (Submitted.)

Brandenburg, A., Nordlund, A., Stein, R. F. \& Torkelsson, U. 1995 Dynamo generated turbulence and large scale magnetic fields in a Keplerian shear flow. Astrophys. J. 446, 741-754.

Brandenburg, A., Nordlund, A., Stein, R. F. \& Torkelsson, U. $1996 a$ The disk accretion rate for dynamo generated turbulence. Astrophys. J. Lett. 458, L45-L48.

Brandenburg, A., Nordlund, Å., Stein, R. F. \& Torkelsson, U. $1996 b$ Dynamo generated turbulence in disks: value and variability of alpha. In Physics of accretion disks: advection, radiation and magnetic fields (ed. S. Kato, S. Inagaki, S. Mineshige \& J. Fukue). Advances in Astronomy and Astrophysics, vol. 2, pp. 285-290. London: Gordon \& Breach.

Brandenburg, A., Saar, S. H. \& Turpin, C. R. 1998 Time evolution of the magnetic activity cycle period. Astrophys. J. Lett. 498, L51-L54.

Brandenburg, A., Dobler, W., Shukurov, A. \& von Rekowski, B. 2000 Collimated outflows from dynamo-active discs. Astrophys. J. (In preparation.)

Covas, E., Tworkowski, A., Brandenburg, A. \& Tavakol, R. 1997 Dynamos with different formulations of a dynamic $\alpha$ effect. Astron. Astrophys. 317, 610-617.

Frisch, U., Pouquet, A., Léorat, J. \& Mazure, A. 1975 Possibility of an inverse cascade of magnetic helicity in hydrodynamic turbulence. J. Fluid Mech. 68, 769-778.

Glatzmaier, G. A. \& Roberts, P. H. 1995 A three-dimensional self-consistent computer simulation of a geomagnetic field reversal. Nature 377, 203-209.

Hawley, J. F., Gammie, C. F. \& Balbus, S. A. 1995 Local three-dimensional magnetohydrodynamic simulations of accretion discs. Astrophys. J. 440, 742-763.

Hawley, J. F., Gammie, C. F. \& Balbus, S. A. 1996 Local three dimensional simulations of an accretion disk hydromagnetic dynamo. Astrophys. J. 464, 690-703.

Hodapp, K.-W. \& Ladd, E. F. 1995 Bipolar jets from extremely young stars observed in molecular hydrogen emission. Astrophys. J. 453, 715-720.

Kleeorin, N. I., Rogachevskii, I. \& Ruzmaikin, A. 1995 Magnitude of the dynamo-generated magnetic field in solar-type convective zones. Astron. Astrophys. 297, 159-167.

Krause, F. \& Rädler, K.-H. 1980 Mean-field magnetohydrodynamics and dynamo theory. Berlin: Akademie (also Oxford: Pergamon).

Matsumoto, R. \& Tajima, T. 1995 Magnetic viscosity by localized shear flow instability in magnetized accretion disks. Astrophys. J. 445, 767-779.

Matsumoto, R., Uchida, Y., Hirose, S., Shibata, K., Hayashi, M. R., Ferrari, A., Bodo, G. \& Norman, C. 1996 Radio jets and the formation of active galaxies: accretion avalanches on the torus by the effect of a large-scale magnetic field. Astrophys. J. 461, 115-126.

Mirabel, I. F. \& Rodriguez, L. F. 1998 Microquasars in our galaxy. Nature 392, 673-676.

Phil. Trans. R. Soc. Lond. A (2000) 
Moffatt, H. K. 1978 Magnetic field generation in electrically conducting fluids. Cambridge University Press.

Ouyed, R. \& Pudritz, R. E. 1997 Numerical simulations of astrophysical jets from Keplerian discs. I. Stationary models. Astrophys. J. 482, 712-732.

Ouyed, R., Pudritz, R. E. \& Stone, J. M. 1997 Episodic jets from black holes and protostars. Nature 385, 409-414.

Parker, E. N. 1979 Cosmical magnetic fields. Oxford: Clarendon.

Pouquet, A., Frisch, U. \& Léorat, J. 1976 Strong MHD helical turbulence and the nonlinear dynamo effect. J. Fluid Mech. 77, 321-354.

Schmitt, D. 1985 Dynamowirkung magnetostrophischer Wellen. PhD thesis, University of Göttingen, Germany.

Shakura, N. I. \& Sunyaev, R. A. 1973 Black holes in binary systems. Observational appearance. Astron. Astrophys. 24, 337-355.

Stone, J. M., Hawley, J. F., Gammie, C. F. \& Balbus, S. A. 1996 Three dimensional magnetohydrodynamical simulations of vertically stratified accretion disks. Astrophys. J. 463, 656-673.

Ustyugova, G. V., Koldoba, A. V., Romanova, M. M., Chechetkin, V. M. \& Lovelace, R. V. E. 1999 Magnetocentrifugally driven winds: comparison of MHD simulations with theory. Astrophys. J. 516, 221-235.

Vainshtein, S. I. 1972 Nonlinear problem of the turbulent dynamo. Sov. Phys. JETP 34, 327331.

Vishniac, E. T. \& Brandenburg, A. 1997 An incoherent $\alpha-\Omega$ dynamo in accretion disks. Astrophys. J. 475, 263-274.

Völk, H. J. \& Atoyan, A. M. 1999 Clusters of galaxies: magnetic fields and nonthermal emission. Astroparticle Phys. 11, 73-82.

Zeldovich, Ya. B., Ruzmaikin, A. A. \& Sokoloff, D. D. 1983 Magnetic fields in astrophysics. New York: Gordon and Breach.

\section{Discussion}

R. E. Pudritz (McCaster University, Canada). The temperatures of protostellar discs around T-Tauri stars are very low as determined by millimetre observations. Also, while T-Tauri stars produce X-rays, this is unlikely to heat the discs to high temperatures. This is the reason that Ouyed and I adopted a cold-wind simulation. In your model, do you maintain the entropy gradient from the disc to the corona throughout your simulation?

A. BRAnDEnBurg. If the turbulence in protostellar discs is really magnetically driven, I would expect that this process leads to significant heating of the corona of the inner parts of the disc, regardless of the temperature at the disc surface. Thus, it seems natural to expect temperatures close to the virial temperature in the corona of the star and the innermost parts of the disc. We did initially hope to get magnetic launching, but in our current setup thermal driving by overpressure seems to be stronger, at least in the inner parts from which the jet emanates. From the outer parts of the disc a slow wind emanates that is crucial for transporting toroidal magnetic field from the disc to the outer parts of the jet. Here the magnetic field angles are indeed favourable for magnetocentrifugal acceleration. Now to your question: yes, we did prescribe the entropy as a function of space. This is done in an attempt to represent some of the physics that is not part of the model. We are currently working on a more self-consistent model where the energy equation is included. 
D. W. Hughes (University of Leeds, UK). The magnetic energy in your simulation of an accretion disc dynamo has not actually saturated, but continues to grow throughout your simulation. Is this not a cause for concern?

A. Brandenburg. I showed results from our first calculations published in our 1995 paper. In that case we did not include any cooling in the disc, so the disc temperature increased by a factor of 10 during the calculation. This is the reason why no statistically stationary state could be expected. In our subsequent calculations we did include volume cooling which did produce a statistically steady state. The resulting butterfly diagram shown in figure 4 is from such a calculation, which we have been carrying out for 300 orbits, and there was no secular variation.

Y. UCHIDA (University of Tokyo, Japan). We have been doing a calculation corresponding to this with a large-scale magnetic field. The large-scale magnetic field plays an essential role to stabilize the system. In your calculation, what stabilizes and keeps the collimated shape of the jets?

A. Brandenburg. Our calculation is only two dimensional, so the $m=1$ kink instability is absent. As Ralph Pudritz points out in his paper (this issue), there is now some evidence from his recent three-dimensional calculations that the strong axial field together with the axial flow contribute to stabilizing the jet. Clearly, we now need to do our calculations in three dimensions. The main purpose of our present two-dimensional calculations was to familiarize ourselves with this new parameter regime. I am not aware of any similar work where the jet is launched and collimated from a dynamo-active disc.

K. Horne (University of St Andrews, UK). Doppler tomography results show that outbursting dwarf nova discs have two-arm spiral shock patterns. Could your local simulations begin to investigate the synergy between spiral waves and the MHD turbulent dynamo? You could impose an epicycle, i.e. a periodic modulation of vertical gravity and radial shear at twice $\Omega_{\mathrm{Kep}}$. I imagine that periodic 'kneading' of the disc could enhance the dynamo, for example, by speeding up conversion of $B_{\phi}$ to $B_{z}$ and $B_{R}$.

A. Brandenburg. At some point we did try to incorporate the effects of density waves, but the problem is that the density wave is not shearing with time, and is therefore not well represented in the shearing-sheet approximation. However, in recent work with Ulf Torkelsson we did look at the effects of an imposed epicyclic modulation and used that to determine the rate of decay of this imposed motion. We did not see any clear enhancement of the dynamo process itself. However, our calculation may not have been long enough and we did not sustain this epicyclic motion. I think we should really turn to global simulations to address problems related to spiral patterns. It is quite possible that a transient spiral wave is a natural consequence of a viscous disc instability.

E. R. PRIEST (University of St Andrews, UK). (i) Can the turbulence produced by the Balbus-Hawley instability in your simulations answer the problem of the origin of the turbulent viscosity required in accretion discs?

(ii) You mentioned doing numerical experiments on shear-induced instabilities for the solar dynamo. How does the resulting dynamo-produced magnetic field vary with latitude and what is its spatial scale? 
A. Brandenburg. (i) The local simulations show that the viscosity alpha depends on the level of the generated magnetic field. An important caveat of local simulations is that the vertical mean field is strictly zero. This is simply a consequence of periodic (or shearing-periodic) boundary conditions in the horizontal directions, which do not allow the vertical flux to change. Thus, if the vertical flux through the box, i.e. the vertical mean field vanishes initially, it remains zero for all times. In a global simulation a significant vertical mean field will be generated, and one must expect that this could enhance considerably the resulting value of the viscosity alpha.

(ii) In our solar convection calculations the spatial scale of the field is comparable with the size of the computational domain. In the present simulations the field does not show overall reversals, however. There are only some vacillations in the mean field whose time-scale is about 10 turnover times. Given our experience with disc calculations we must expect that the properties of the large-scale field are strongly controlled by boundary conditions and by the geometry of the simulation domain. This underlines the urgent need for global simulations. I should also mention that in the local convection simulations the dynamo alpha is again very noisy, but that its mean value is small and positive in the northern hemisphere.

S. FAlle (University of Leeds, UK). Have you done any tests to determine how realistic it is to restrict the domain of your simulations of dynamo action in accretion discs to a small box? One could for example easily do a two-dimensional calculation of the whole disc and then compare this with one in which the computational domain is a box of the same relative size as the one used in the three-dimensional calculation.

A. Brandenburg. In our accretion disc simulations we have adopted the shearing sheet approximation, which is strictly valid only at infinitely large radii. One can however restore certain $1 / R$ factors in order to apply the simulations to a finite radius and, in particular, to remove the ambiguity between inward and outward flows. There is now a global simulation by Philip Armitage (Armitage 1998), where a certain radial range is covered, but the vertical dependence is modified to remove vertical density stratification. Understandably, no large-scale field is found (this requires an alpha effect, which, in turn, requires stratification), but the small-scale dynamo activity is otherwise similar to that in our simulations. Also, the Shakura-Sunyaev viscosity alpha has similar average values to those found by us and others using local simulations. 